\title{
RoboCup Humanoid Challenge
}

\author{
Minoru Asada ${ }^{\# * 1}$ and Norbert Michael Mayer ${ }^{\# 2}$ \\ * Department of Adaptive Machine Systems, \\ Graduate School of Engineering, Osaka University, \\ 2-1 Yamada-oka, Suita, Osaka 565-0871, Japan \\ 1 asada@ams.eng.osaka-u.ac.jp \\ \#Asada Synergistic Intelligence Project, ERATO JST, \\ 2-1 Yamada-oka, Suita, Osaka 565-0871, Japan \\ ${ }^{2}$ michaele jeap.org
}

\begin{abstract}
We describe here the project of the humanoid challenge that is part of the RoboCup robot soccer competitions. We focus on how the relevant research issues of humanoid robotics -for example biped walking and human-like sensors and actuators- can be addressed and we investigate how the teams proceed to solve the given tasks. Thus, new technologies like artificial muscles and artificial skin might find its way into the competition very soon. We go into details of examples of these technologies and discuss in which way they may contribute to the RoboCup and in return how the RoboCup may serve as a benchmark for achievements within these technologies. Further, we describe how the RoboCup works as an open, worldwide cooperative project in robotics and AI.
\end{abstract}

\section{INTRODUCTION}

RoboCup is an attempt to foster intelligent robotics research by providing a standard problem of which the ultimate goal is to build a team of eleven humanoid robots that can win against the human world cup champion soccer team by 2050 . It's obvious that building a robot to play the game of soccer is an immense challenge. RoboCup is designed as a vehicle to promote robotics and AI research, by offering a publicly appealing but formidable challenge [16], [4].

A unique feature of RoboCup is that it is a systematic attempt to promote research using a common domain, mainly soccer. Also, it is perhaps the first to explicitly claim that the ultimate goal is to win against the human world cup champion team. Building a robot to play soccer would certainly be considered as a major achievement in the field of robotics, and numerous technology spin-off can be expected during the course of the project. We call this kind of project a landmark project, and RoboCup is definitely a project of this kind.

Since the first RoboCup in 1997 [17], it has grown into an international joint-research project in which about 4000 researchers from 40 nations and regions around world participate (see Figure 1), and it is one of the most ambitious landmark projects of the 21 st century. RoboCup currently consists of three divisions: RoboCup Soccer aiming towards the final goal, RoboCupRescue, a serious social application to the rescue activities for any kind of disasters, and RoboCupJunior, an international education-based initiative designed to introduce young students to robotics. Recently, RoboCup@home was added to promote daily life applications from technology advances fostered by the other existing RoboCup divisions, and some experimental demonstrations were also shown in RoboCup 2007 towards the future official leagues. For more details, please refer to [23], [6], [9], [25], [27], [3], [28], [26], [7], [14], [1], [10].

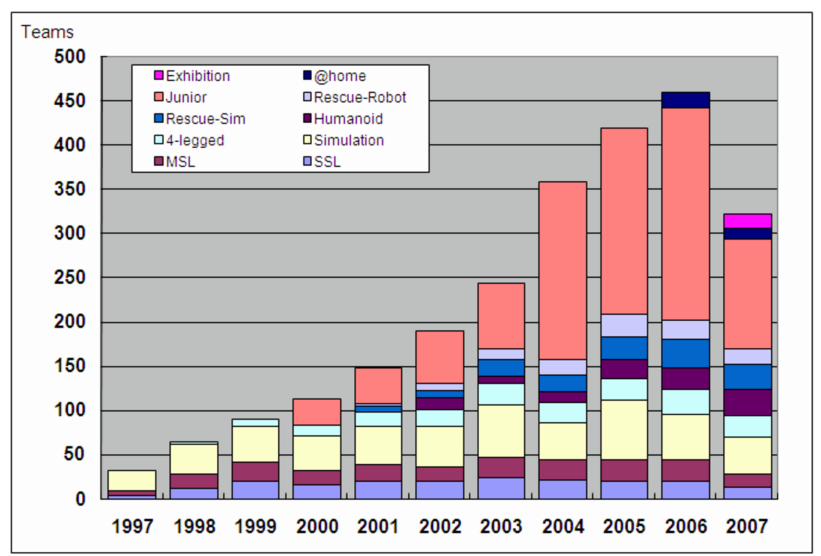

Fig. 1. The number of teams

In RoboCup 2007 that was held at the Georgia Institute of Technology, Atlanta, USA, the first match between a human team (the trustees of the RoboCup Federation) and the MSL champion team (Brainstormers Tribots Univ. of Osnabrueck) has been demonstrated. Figure 2 shows a picture during the match. Unfortunately, the robot team lost the game, but the match itself was much of fun and new research issues related to human-robot interactions were found.

While the first RoboCup was held in $1997^{1}$, the Humanoid League (HL) has no sooner been established than 2002 at the RoboCup in Fukuoka [5]. The reason for this in comparison to other RoboCup soccer leagues relatively late start is presumably that biped walking was and partly still is a challenge in robotics. However, during the last some years better and better solutions to this problem have been found, are presented and tried out at the RoboCup. Following the trace from the first competition in 2002 one can see how closely the RoboCup follows the state of the art. For example, the Best Humanoid

\footnotetext{
${ }^{1}$ Actually, pre-RoboCup was held in 1996 in Osaka in conjunction with IROS 1996 Osaka.
} 


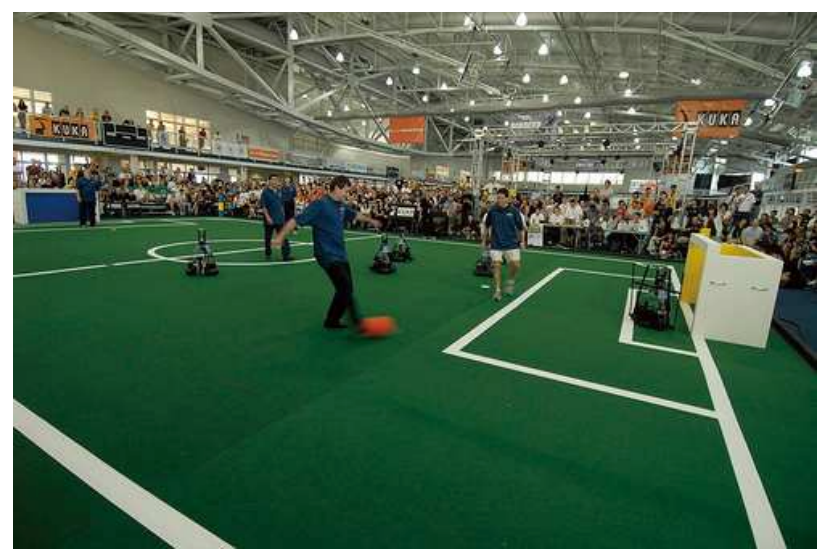

Fig. 2. The first match between human team and MSL robots (through the courtesy of Prof. Tucker Balch, the general chair of RoboCup 2007)

\begin{tabular}{|l|l|}
\hline Year & \\
2002 & Start of HL \\
& 3 size classes $40 \mathrm{~cm}, 80 \mathrm{~cm}, 120 \mathrm{~cm}$ \\
& 3 competitions \\
\hline 2005 & self-contained robots \\
& $2-2$ games replace the free style competition \\
& 2 size classes: KidSize and TeenSize \\
\hline 2007 & Humanoid robots in 3D SocSim \\
\hline
\end{tabular}

TABLE I

OVERVIEW OF THE ROBOCUP HL HISTORY

of the RoboCup 2003 in Padova was a platform based on a prototype of the Honda Asimo robot. At that time the Honda Asimo has been seen as by far the best and most advanced humanoid robot. Since the start the HL underwent a profound development. Competitions and challenges have changed in various ways; rules matured in many points and gained more focus on the issues that are essential from a technical point of view; and of course the robots became better. At the moment the focus has been shifted to smaller robots for several reasons which we are going to point out below.

The most important aspect from a viewpoint of the RoboCup project is to provide the appropriate environment to address the current research issues in general and the research issues of humanoid robotics and biped walking in particular with respect to the HL. At the moment the most interesting research issues are to encourage dynamic motions - in particular walking - and human-like sensors. In this paper we discuss these issues and outline in how we intend to design the rules in order to encourage the research in the best way.

The rest of the paper is organized as follows. First, the brief history of the league is described. Next, the future issues not restricted to HL but to humanoid research in general are given.

\section{History of the Humanoid League}

In the first years (2002-2004) the robots that participated in the HL were quite variant in many respects and had to be sorted into three sub-leagues in order to cope with the variety of heights ranging from $10 \mathrm{~cm}$ to over $2 \mathrm{~m}$ (see Figure

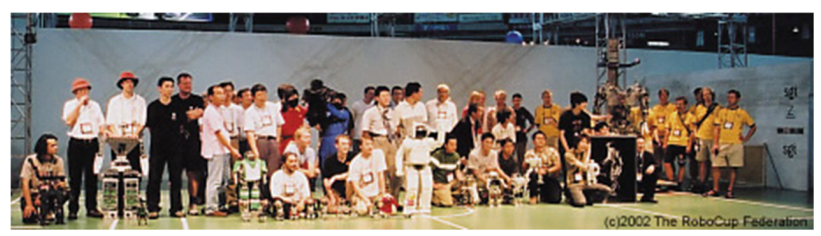

Fig. 3. The first HL robots and team members in RoboCup 2002

3). The competitions consisted of walking challenges, a free style competition, and penalty kick competition for all size classes. At that time external processing - even remote control - was allowed. In order to make results of the competitions comparable between the very different robots, the performance factors had been introduced. These performance factors had to be applied to commercial platforms, remote control and external processing.

The emergence of Team Osaka in 2004 in Lisbon showed an unprecedented performance with regard to technical compactness and general perfection in their size class and in relation to the manufacturing costs. They got the Best Humanoid Award in that year. At that time their robot arose some hope that regular soccer games were indeed possible with robots of a size of roughly $40-60 \mathrm{~cm}$ and certain design features. These features have hitherto been adopted by most teams of the later established KidSize class.

Starting from these experiences many changes have been introduced into the competition of the year 2005 making the technical constraints more specific. Performance factors were abandoned, and external processing as well as remote control were banned from the competition. Thus, the processing of sensory information, behavior processing, etc. has to be completely self-contained within the platform. A maximum ratio between foot size and height of the center of mass had been introduced in order to encourage dynamic walking. The number of size classes was reduced from 3 to 2, of which the smaller class was called Kidsize $(<60 \mathrm{~cm})$ and the bigger class TeenSize. The total number of competitions remained the same, however, the free style competition was replaced by the regular 2 on 2 games in the KidSize class. In the TeenSize class the conductance of 1-1 games was discussed, but could not be carried out. One aim of the technical committee was and still is to lead the development towards current research problems. Dynamic walking and stability have been the most important issues then and still are up to now, which have been enforced by the technical challenges between the years 2005 and 2006 . In the years 2005 and 2006, a rough terrain challenge has been conducted where the robots have to cross over a field of hexagonal tiles, which are of a random height. The technical challenges change every year.

The rules have been further refined for the competitions in 2006 and 2007 in many aspects, in particular with respect to the conductance of the 2 on 2 games. Also the footrace competition had been introduced to the TeenSize class in order to have an equal number of competitions in Teen- and KidSize.

The rules of 2005 and 2006 and the example of relatively 
cheap and powerful robots gave a new perspective to many interested people in the RoboCup community and also people from outside who were interested in setting up a team.

In 2005 a total of 20 teams from 9 nations and regions participated. This is about twice the number of the year 2004 . For the first time a qualification process had to be introduced. Several teams had some background from other leagues and took the advantage to customize their software relatively successfully within the new league. Team Osaka got the Best Humanoid Award again, as well as in 2006. At the RoboCup 2007 in Atlanta a total of 29 teams participated, of which were 22 from KidSize and 7 from TeenSize. The technical level of the participating teams increased significantly. Although the finals of the 2 on 2 games in KidSize were won by team NimbRo for the first time, due to a better performance in the Technical Challenge Team Osaka was again able to win the Best Humanoid Award.

In the following section we want to outline the evolutionary process and describe a kind of typical robot of the HL VisiON TRYZ (used by Team Osaka and JEAP). Then we describe further plans of the HL that are currently under discussion.

\section{EVOLUTIONARY CONVERSION AND CURRENT TYPICAL ROBOTS}

In the first years quite a variety of different types of humanoid robots participated. Fig. 4, upper half, shows the histograms over the heights of the participating robots in 2002 - the first year of the Humanoid League, and 2005 - which was the first year of the 2 on 2 competitions. In each graph, the plotted Gaussian distribution shows the same mean, variance as the data-set of the respective histogram. In the top pair of graphs one can see the histogram of heights of all robots that participated in 2002 and 2005. Robots of the year 2005 showed a significantly smaller variance in size than the robots that participated in the first year of the Humanoid League ${ }^{2}$.

Using only this one parameter one can clearly see a developmental and convergence process towards robots of sizes between 40-60 cm. Also, more and more robots participating in the RoboCup Humanoid League are exclusively manufactured for this event. The convergence is partly caused by the rules in the KidSize class that allows a maximum height exactly at the size of $60 \mathrm{~cm}$, but mainly it is due to constraints that come with considerations of the mechanical design and costs. The convergence process happens mainly in the KidSize class, where the typical design concept of the robots' hardware consists of the following parts

- Servo motors (initially designed for RC toys). In particular many teams switched to RC servos that can be linked together in the RS 485 bus (similar to the well known RS 232; one example are Dynamixel DX 117 and AX 12 actuators).

- Small reliable mini PCs (e.g. handheld computers, industry one board mini PCs, like PINON PNM SG3F). In

\footnotetext{
${ }^{2}$ Only robots were counted that showed any kind of movement during the competition.
}

order to process the vision stream of about 15 frames at a resolution of $640 \times 480$ a $600-800 \mathrm{MHz}$ processor is sufficient.

- Micro-controller, these are necessary for the real time control of the servos.

- As sensors: camera (connected via USB or Fire-wire to the PC) and attitude sensors (gyro, acceleration sensors). Except for the feedback from the joint angles most robots do not use additional sensors.

- Wireless network (IEEE 802.11) is permitted, and can be used for the communication between the robots and in order to send start and stop signals to the robots. However, wireless networks are not reliable during the RoboCup. A fallback solution is highly recommended. The rules state that the robot has to be able to perform even if the wireless network is not working.

Whereas the KidSize robots evolved rapidly during the past 2-3 years, we expect the same development in the TeenSize yet to come. Typically, TeenSize robots are either derived from KidSize models (typically just on the lower limit of the permitted size of the TeenSize class) or we see that robots participate from initially unrelated fields of research. It is very much to hope that in the near future a TeenSize class with its own profile and own technology evolves.

For this purpose a gap of size has been introduced between TeenSize class and KidSize class. This gap has been increased from initially $5 \mathrm{~cm}$ (2006) to $20 \mathrm{~cm}$ (2007) and is going to be increased further to $40 \mathrm{~cm}$ (2008).

The RoboCup 2007 indicated a first positive development of the TeenSize class. The lower half of Fig. 4 shows the histograms of the heights of the robots in TeenSize and KidSize. In the TeenSize class 7 teams with a total of 12 different robot types participated. One can see that most robots have a height on the lower end of the permitted height span. The variance is still high.

The histogram of the KidSize class shows a rather narrow height distribution and a further increase of the number to almost the maximal value of 24 teams. More teams are hard to organize within one competition, and also normally not permitted by the local organizers. In the TeenSize class a wide variety of technological solutions have been presented and as of the state of 2007 no standard has been established.

In the following we describe the current and previously used robot VisiON TRYZ and VisiOn 4G (Team JEAP [21] and Team Osaka[18]) in more detail, which - with respect to the criteria outlined above - can be seen as typical robots of the KidSize class. We also briefly outline a typical software environment. The body structure of the $4 \mathrm{G}$ is aluminium whereas the body of the previous TRYZ robot was made of carbon fiber reinforced plastic (CFRP), the motors are RC servos manufactured by the robot's manufacturer. The motors can be connected in series in a proprietary bus hardware that bases on the RS 232 serial bus. Their functional design is similar to the above mentioned Dynamixel servos. The still experimental servos of the VisiON TRYZ have a plastic chassis the newer servos in the $4 \mathrm{G}$ are made of aluminium, 

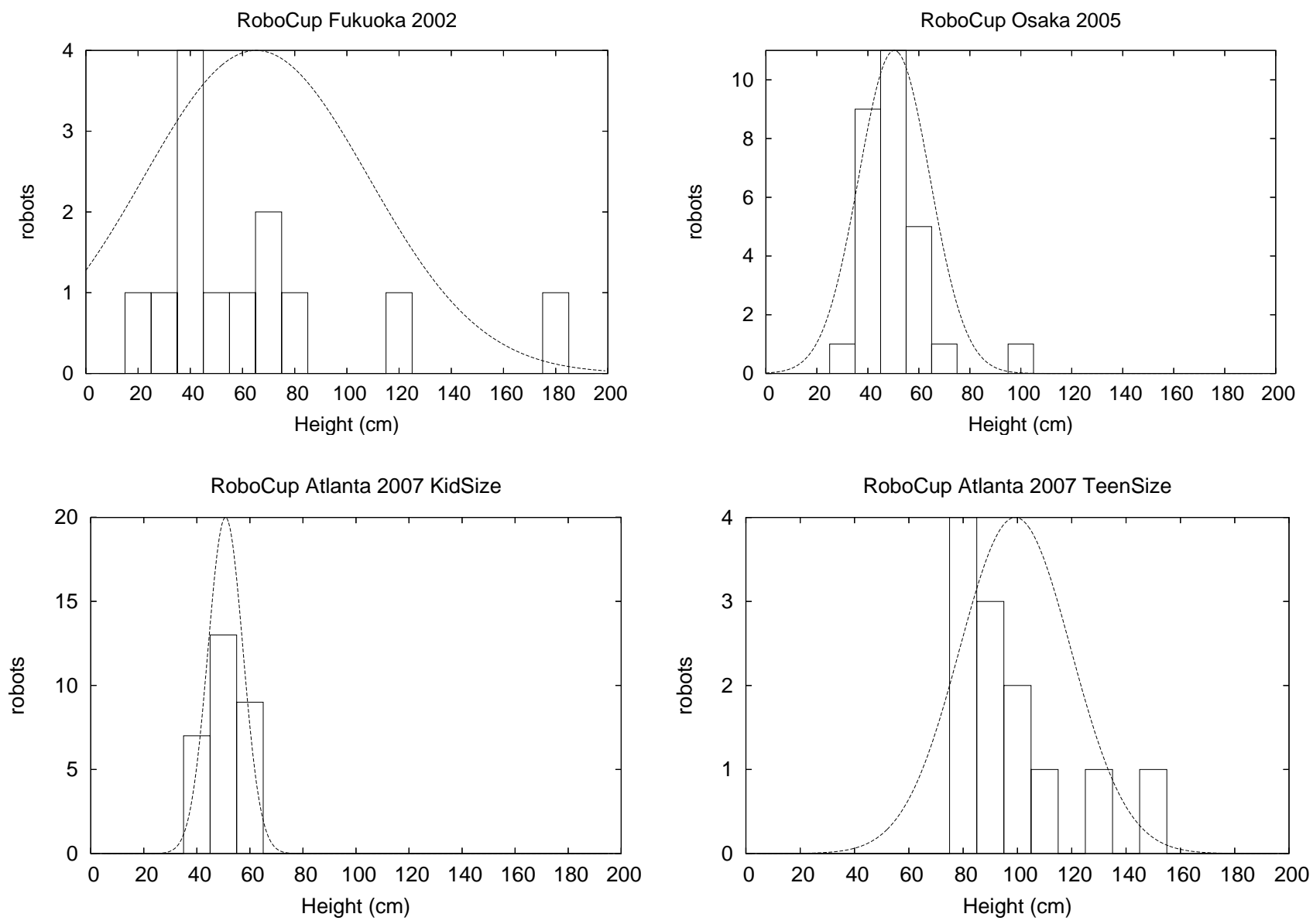

Fig. 4. Histograms of the heights of robots that participated at several HL competitions between 2002 and 2007. The upper line shows the histograms of the heights of the robots in the competitions in the year 2002 and 2005. The lower line shows the histograms of the year 2007's KidSize class $(<60 \mathrm{~cm})$ and TeenSize class $(>80 \mathrm{~cm})$ class separately.

which results in a significantly higher robustness and extended lifespan. The foot size of the VisiON $4 \mathrm{G}$ robot became smaller and thus easily complies with this year's (2007) rules. In the $4 \mathrm{G}$ robot the number of DOF is reduced to 22 (from 26 in the TRYZ robot). The system of a camera and other sensors such as acceleration sensors are similar to the TRYZ robot, although the bus system utilized in the $4 \mathrm{G}$ is rather sophisticated. The $4 \mathrm{G}$ internally uses three different serial buses: Two customized RS 232 and an I2C, which is a challenge for the real time paradigm. Theoretically also a USB bus can be used for the communication between the mini PC and the motor controller. The I2C-bus serves for the communication between the acceleration and gyro sensors and the motor controller.

\section{THE CURRENT STATUS AND NEAR FUTURE RESEARCH ISSUES}

The two size classes, TeenSize and KidSize, in the Humanoid League are separated basically by the height $H$ of the robots. In order to have a sound definition, currently the height of a robot is defined as

$$
H=\min \left\{H_{\text {top }}, 2.2 \times H_{\text {com }}\right\},
$$

\begin{tabular}{|c|c|c|c|c|}
\hline \multicolumn{3}{|c|}{ VisiON TRYZ } & \multicolumn{2}{|c|}{ VisiON 4G } \\
\hline Height (mm) & \multicolumn{2}{|c|}{475} & \multicolumn{2}{|c|}{445} \\
\hline Weight (kg) & \multicolumn{2}{|c|}{3.1} & \multirow{2}{*}{\multicolumn{2}{|c|}{$\begin{array}{l}3.2 \\
22\end{array}$}} \\
\hline $\mathrm{DOF}$ & \multirow{2}{*}{\multicolumn{2}{|c|}{$\begin{array}{c}26 \\
\text { VStone Servo }\end{array}$}} & & \\
\hline Actuators & & & \multicolumn{2}{|c|}{ VStone Servo } \\
\hline Camera Type & \multicolumn{2}{|c|}{ Quickcam } & \multicolumn{2}{|c|}{ Quickcam } \\
\hline Controller & Main Controller & Sub controller & Main Controller & Sub controller \\
\hline CPU & Geode LX 800 & SH2 F7054F & PNM-SG3 & ARM \\
\hline ROM & 4GB (Flash HDD) & $384 \mathrm{~KB}$ & 4GB (CF Flash) & $512 \mathrm{~KB}$ \\
\hline RAM & $512 \mathrm{MB}$ & $64 \mathrm{~KB}$ & $512 \mathrm{MB}$ & $40 \mathrm{~KB}$ \\
\hline
\end{tabular}

TABLE II

VISION TRYZ AND VISION 4G HARDWARE SPECIFICATIONS

where $H_{\text {top }}$ is the actual height and $H_{\text {com }}$ is the height of the center of mass of the robot.

The aim is that two different sets of research issues are addressed in the two leagues, on the basis of what is technically possible currently.

Due to the size and the weight constraints, the KidSize class has advantage to the TeenSize class in applying the commercially available parts such as motor controller and sensor units. The walking behavior in this class is much more stable than the TeenSize, and passing and shooting behaviors are realized in many teams. Therefore, the current research 


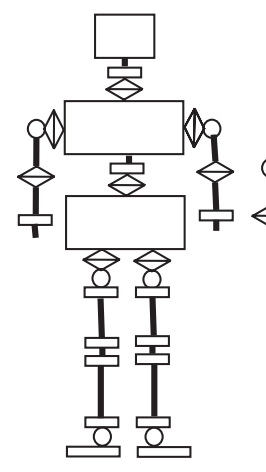

VisiON TRYZ

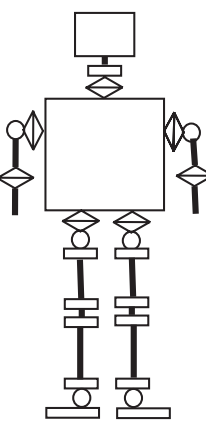

VisiON 4G

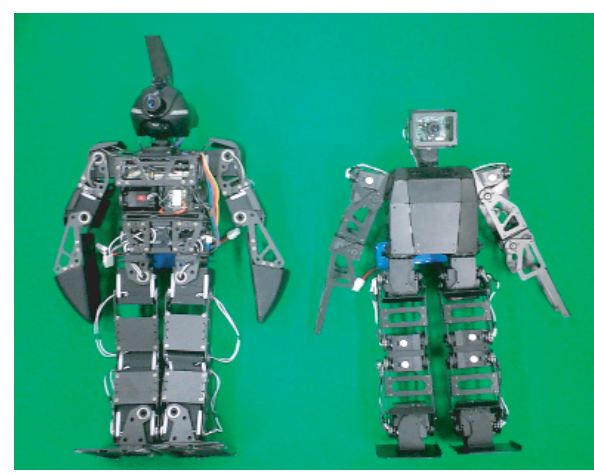

VisiON TRYZ

VisiON 4G

Fig. 5. The VisiON TRYZ and VisiON 4G robot: On the right side, a photo from the front view of the robots is depicted. One can see the camera (Philips chip-set) and the USB connectors for servicing in the head of the robot. On the left side is a schematic overview of the actuators and their attitude in relation to the bodies.

issues are action planning and team coordination. For this reason it seems preferable to coordinate activities with other leagues such as the Standard Platform League that will be introduced in 2008 instead of the 4-Legged league, and the 3D Soccer Simulation League.

Currently, the Humanoid League allows to use the omnidirectional vision system to capture the whole scene. The next challenge is to use the normal, perspective vision system much similar to human vision system.

For the TeenSize class, the suggestion of the authors would set the focus more on issues like experimental actuators, motor skill, human machine interaction. The number of participants in the TeenSize class is much smaller than in the KidSize class. Beside the cost issue, the reason seems that the motor controller in this class is not commercially available yet although this class is closer to the final goal than the KidSize class. Thus motor controller units have to be designed by the teams themselves.

The common research issues are vision and cognition. It is intended to permit only human-like sensors. For 2008 it is planned to reduce the allowed visual field of all cameras to 180 degrees. In particular omnivision cameras are going to be banned.

One classic field of RoboCup is cognition and team strategy. At present team strategy is of minor importance in comparison to other factors in the competition. This could change within the next several years. The following changes could provide the right environment for the team strategy.

- Increase the number of players. This has been a very emotional discussion in the past years, because the costs increase significantly with each additional player. Various test games of mixed teams have been conducted during the previous RoboCup competitions. At the moment, we are planning to increase the number of players. The number of players in each team is going to be increased from 2 to 3 in the competition in 2008.

- Closer collaborations with other RoboCup leagues that already have a better developed culture of team strat-

\begin{tabular}{|c|l|}
\hline Year & \\
\hline 2008 & $\begin{array}{l}\text { 3-3 Games } \\
\text { Omnivision banned } \\
\text { Start of standard platform league }\end{array}$ \\
\hline 2010 & $\begin{array}{l}5-5 \text { Games in KidSize } \\
\text { 3D2Real } \\
\text { no color keying }\end{array}$ \\
\hline 2025 & $\begin{array}{l}\text { start of full size league } 150-200 \mathrm{~cm} \text { games } \\
\text { real human-like sensors like skin etc. } \\
\text { robots interact directly with referees } \\
\text { team play }\end{array}$ \\
\hline
\end{tabular}

TABLE III

ROAD MAP FOR THE HUMANOID LEAGUE AND RELATED LEAGUES WITHIN THE ROBOCUP COMPETITION.

egy and cognition. The optimization of the interaction between the leagues is going to be an important issue within the next several years in particular since additional leagues turn towards humanoid robotics. Currently this step has been done in the 3D Soccer Simulation League (SocSimL) which since 2007 simulates humanoid robots and the former four legged league which is going to become now the "Standard Platform League" that is using a standard humanoid robot.

In the table III the road map of the humanoid league in near future is depicted.

\section{MORE FUNDAMENTAL RESEARCH ISSUES IN FUTURE HUMANOID ROBOTICS}

In this section, we review the future research issues described in [15], especially focusing on the recent progress. With regard to human-like sensors and actuators the the recently introduced humanoid "Child-robot with Bio mimetic Body $\left(C B^{2}\right)$ " for cognitive developmental robotics developed by JST ERATO Asada Project [2] may serve as an example although it cannot walk.

$C B^{2}$ has a whole-body soft skin (silicon surface with 197 high sensitive tactile sensors underneath) and flexible joints (51 pneumatic actuators). The height and the weight are $1.3 \mathrm{~m}$ 
and $33 \mathrm{~kg}$, respectively. It also has two eyes, two ears, and a vocalization unit (a simple version of the vocal system used in [29]). Figure 6 shows pictures of $C B^{2}$, where the left one indicates its whole body and the bottom right ones indicate its mechanical structure and the attachment of 197 tactile sensors underneath of the whole body silicon surface.

\section{A. Surface Materials and Tactile Sensing}

Towards the final goal, security becomes more and more important since we have to deal with intensive robot-human interaction. Thus, the humanoid robots at that stage must have soft surface materials in order to avoid damages and injuries. However, at the same time, a mechanism is needed that protects internal mechanics and electronics from external force. The competitions do not include any human players yet. Still, the TeenSize class robots require a more serious consideration of these issues. Currently, hard plastics or metals are usually used as the surface materials of the robots. Damages caused by falling down or any collisions with other robots or objects in the KidSize class are not so serious as that of the TeenSize class. Therefore, it has been suggested that the human assistants should be allowed to stay closer to the TeenSize robots than in the TeenSize class. In the KidSize class where the human assistants are not allowed to stay on the playground during the match. So, in the next several years one would expect a more serious discussion about safety in human-robot interaction at least in the TeenSize class.

Silicon materials are often used as the soft skin of robots, and $C B^{2}$ has a whole body silicon surface to realize the soft skin considering the physical interactions with humans. In the future, such soft skin is necessary for RoboCup humanoid players to interact with human ones. At the same time, tactile sensing is a very important perception to detect collision (or to feel pain). In the case of $C B^{2}, 197$ PVDF (polyvinylidene fluoride) units are used for tactile sensing. They are attached between the surface silicon skin. Additional sponge rubbers absorb the external force to protect the internal machinery. Recently, a super-flexible sensor system has been developed for humanoid robots which comes together with another type of artificial skin [24]. The skin has elastic properties and is at the same time capable of tactile sensing without any structured sensor harness in or underneath the sensing area. It could turn out to be very useful if it would be applied in the larger robots, say in the TeenSize class.

Anthropomorphic fingertips with multi-modal sensors have been developed for human-like hand perception with fingers and palm[13]. The fingertip consists of two silicon rubber layers of different hardness containing two kinds of receptors, strain gauges and PVDF films distributed randomly as receptors. In the future these technologies can be used for the hands of goalies to keep the goal or that of players to throw the ball in the field.

a) Actuators and Mechanical Design: In general, electric motors are widely used for humanoid behavior generation, and in the RoboCup humanoid league, many teams are utilizing RC servo motors to generate walking and ball kicking behaviors.
The electrical motors and reduction gears play a great role to achieve high-performance trajectory tracking, but on the other hand, it is relatively difficult to realize joint compliance by utilizing them. As a result, such a robot that consists of electrical motors and gears is not suitable for studying dynamic whole body motions such as running, dribbling, jumping, heading, and so on. Toward the generation of such motions, anthropomorphic biped walkers driven by antagonistic McKibben artificial muscles have been developed [12]. these robots have shown jumping and running behaviors though these behaviors are still difficult to be stabilized. $C B^{2}$ adopts another pneumatic actuator, that is, air cylinder type to generate elastic motions. These pneumatic actuators have no backlash, however, due to the compressible fluid property, it is hard to realize high performance of responsibility and accurate position control. Recently, a linear new actuator with long stroke, high response, and large thrust using a Halbach array of magnets is developed and analyzed [22]. This seems promising for future humanoid actuators owing to preferable features such as high responsibility, high power, robustness against dynamic motions due to the lack of gear backlash, and so on.

Currently, most robotic joints adopt one DoF type and therefore one DoF actuators are used. Sometimes, more DoFs joints are devised, but with a set of several one DoF actuators. Recently, a spherical resonant actuator was proposed as a multi-joint with a multi-DoF actuator [11]. These sorts of actuators are intended to be used at shoulders, wrist and ankles of humanoids. Figure 7 shows the basic structure of these devices.

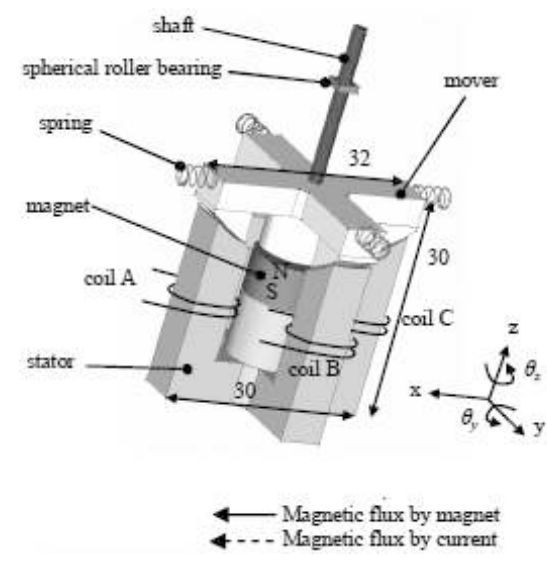

Fig. 7. Basic structure of the proposed model of the spherical resonant actuator (through the courtesy of the authors from the reference [11])

\section{DisCUSSION}

In this overview we intended to describe which current research topics can be addressed in the RoboCup Humanoid League. In this way the HL can be forum of discussion for interested researchers. It is also a benchmark to test new technologies under rough conditions for their applicability with respect to robotics and AI. Further we see the HL 


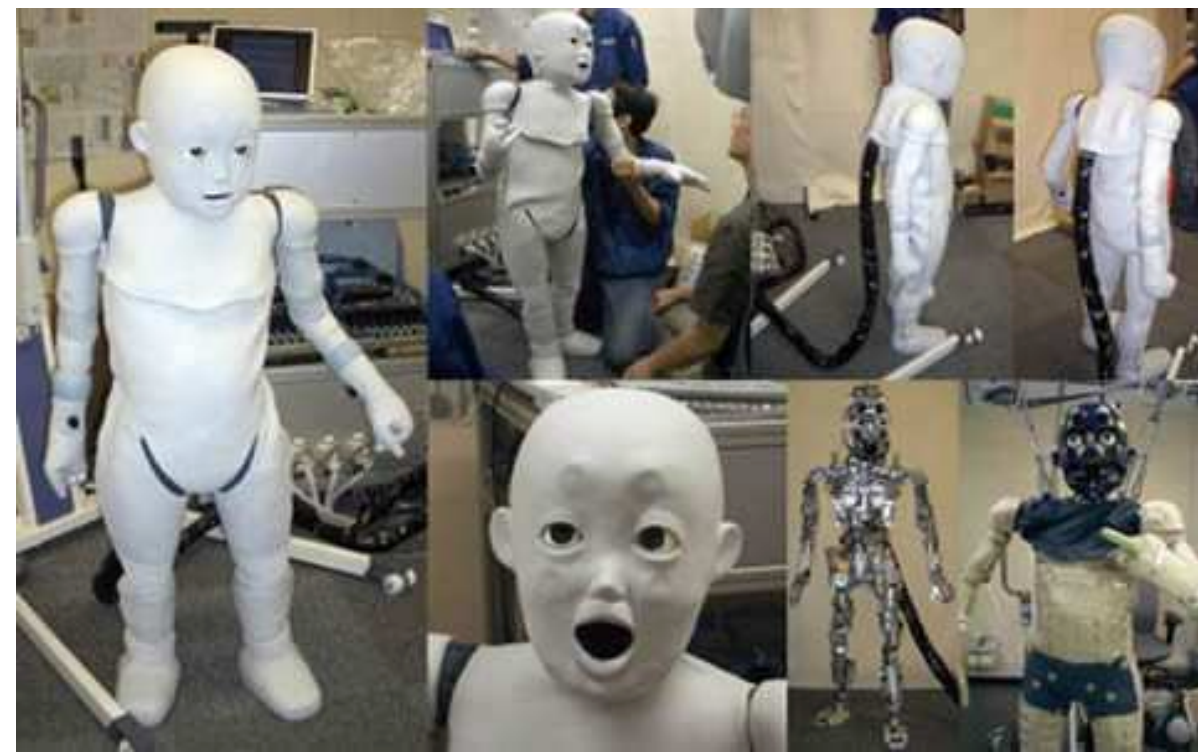

Fig. 6. A child-robot with Bio mimetic Body $\left(C B^{2}\right)$ toward a developmental robot through interaction with humans (from http://www.jeap.org/web/pressrelease.html)

as an educational tool that can help to bring students into intensive contact with problems and methods of robotics. Also, the media interest during the last 2 years ahs been a great help in this respect. For example, the finals of the Humanoid League were covered as live television events. Thus, Humanoid League can be a nice way to present research to a broad audience.

One important challenge from point of the organization is how the activities of the new leagues that deal with humanoid robots (3D Soccer Simulation League, Standard Platform League) can be integrated into an overall concept. One has to see how the development in these leagues evolves and the what specific research topics are going to turn out to be the most interesting.

One initial step towards the coordination of the 3D Soccer Simulation League and the Humanoid League is the 3D2Real project[20], [8], [19]. The aim is here to port the tactical know how of the simulation league to real humanoid robot platforms and to test how synergies of the two different leagues can be used for the benefit of the RoboCup project and the year 2050 goal. The formal target is to conduct the finals of the 3D simulation league some day in real robots, and thus show then to be virtues of simulation. Part of the project is a suggested environment that makes participants' behaviors applicable to both the simulation and the real robots. The intention is of course that the behavior is identical in both cases. Research issues there are going to be which sensor statistics are realistic and what kind of physical simulation is sufficient to give realistic results.

All in all we see a rapid change in the structure of the soccer playing leagues towards humanoid robots. Assuming the enormous energy effort that is usually invested into the competition by the participants it is to expect that these new humanoid leagues are going to evolve in similar way like the Humanoid League.

\section{ACKNOWLEDGEMENT}

We thank the other members of the TC for vivid discussions during the last some years, Joschka Boedecker for cross-reading this article. N. M. thanks Takashi Minato for discussions with regard to the SB2. Thanks go also to Jackie Baltes for digging out important data!

\section{REFERENCES}

[1] Brett Browning Andrea Bonarini, Daniel Polani and Kazuo Yoshida, editors. RoboCup 2003: Robot Soccer World Cup VII. Springer, Lecture Note in Artificial Intelligence 3020, 2004.

[2] Minoru Asada, Koh Hosoda, Yasuo Kunioshi, Hiroshi Ishiguro, Toshio Inui, and Ken Nishimura. Synergistic intelligence: Approach to human intelligence through understanding and design of cognitive development. In The 5th International Conference on Development and Learning (ICDL'06), 2006.

[3] Minoru Asada and Hiroaki Kitano, editors. RoboCup-98: Robot Soccer World Cup II. Springer, Lecture Note in Artificial Intelligence 1604, 1999.

[4] Minoru Asada, Hiroaki Kitano, Itsuki Noda, and Manuela Veloso. Robocup: Today and tomorrow - what we have learned. Artificial Intelligence, 110:193-214, 1999.

[5] Minoru Asada, Oliver Obst, Daniel Polani, Brett Browning, Andrea Bonarini, Masahiro Fujita, Thomas Christaller, Tomoichi Takahashi, Satoshi Tadokoro, Elizabeth Sklar, and Gal A. Kaminka. An Overview of RoboCup-2002 Fukuoka/Busan. AI magazine, 24(2):21-40, 2003.

[6] Minoru Asada, Manuela M. Veloso, Milind Tambe, Itsuki Noda, Hiroaki Kitano, and Gerhard K. Kraetzschmar. Overview of robocup-98. AI magazine, 21(1):9-19, 2000.

[7] Andreas Birk, Silvia Coradeschi, and Satoshi Tadokoro, editors. RoboCup 2001: Robot Soccer World Cup V. Springer, Lecture Note in Artificial Intelligence 2377, 2002.

[8] Joschka Boedecker, Norbert Michael Mayer, Masaki Ogino, Rodrigo da Silva Guerra, Masaaki Kikuchi, and Minoru Asada. Getting closer: How Simulation and Humanoid League can benefit from each other. In Proceedings of the 3rd International Symposium on Autonomous Minirobots for Research and Edutainment (AMiRE), 2005. 
[9] Silvia Coradeschi, Lars Karlsson, Peter Stone, Tucker Balch, Gerhard Kraetzschmar, and Minoru Asada. Overview of robocup-99. AI magazine, 21(3):11-18, 2000.

[10] C. Sammut J. Santos-Victor D. Nardi, M. Riedmiller, editor. RoboCup 2004: Robot Soccer World Cup VIII. Springer, Lecture Note in Artificial Intelligence 3276, 2005.

[11] Y. Hasegawa, T. Yamamoto, K. Hirata, Y. Mitsutake, and T. Ota. New spherical resonant actuator. In ISEF 2007 - XIII International Symposium on Electromagnetic Fields in Mechatronics, Electrical and Electronic Engineering.

[12] Koh Hosoda and Kenichi Narioka. Synergistic 3d limit cycle walking of an anthropomorphic biped robot. In Proceedings of IEEE/RSJ International Conference on Intelligent Robotics and System (IROS) 2007), 2007. Vol.CD-ROM.

[13] Koh Hosoda, Yasunori Tada, and Minoru Asada. Anthropomorphic robotic soft fingertip with randomly distributed receptors. Robotics and Autonomous System, 54(2):104-109, 2006.

[14] Gal Kaminka, Pedro U. Lima, and Raul Rojas, editors. RoboCup 2002: Robot Soccer World Cup VI. Springer, Lecture Note in Artificial Intelligence 2752, 2003.

[15] H. Kitano and M. Asada. The robocup humanoid challenge as the millennium challenge for advanced robotics. Advanced Robotics The international Journal of the Robotics Society of Japan, 13:723-736, 2000.

[16] H. Kitano, M. Asada, Y. Kuniyoshi, I. Noda, E. Osawa, and H. Matsubara. "robocup: A challenge problem of ai". AI magazine, 18(1):73-85, 1997.

[17] Hiroaki Kitano, editor. RoboCup-97: Robot Soccer World Cup I. Springer, Lecture Note in Artificial Intelligence 1395, 1998.

[18] Reo Matsumura, Naoki Shibatani, Takuro Imagawa, Takeshi Maeda Takahiro Miyashita, Tomotaka Takahashi, Yohei Akazawa, Nobuo Yamato, and Hiroshi Ishiguro. TeamOsaka (Kid Size) Team Description Paper. In RoboCup 2007 Symposium papers and team description papers CD-ROM, 2007.

[19] N. Michael Mayer, Joschka Boedecker, Kazuhiro Masui, and Minoru Asada. HMDP: A new protocol for motion pattern generation towards behavior abstraction. In RoboCup Symposium, 2007. Proceedings CD Rom.

[20] Norbert Michael Mayer, Joschka Boedecker, Rodrigo da Silva Guerra Oliver Obst, and Minoru Asada. 3D2Real: Simulation league finals in real robots. In Gerhard Lakemeyer, Elizabeth Sklar, Domenico G. Sorrenti, and Tomoichi Takahashi, editors, RoboCup 2006: Robot Soccer World Cup X, Lecture Notes in Artificial Intelligence, pages 25-34. Springer, 2006.

[21] Norbert Michael Mayer, Masaki Ogino, Rodrigo da Silva Guerra, Joschka Boedecker, Sawa Fuke, Hiroyuki Toyama, Ayako Watanabe, Kazuhiro Masui, and Minoru Asada. JEAP Team Description. In RoboCup 2006 Symposium papers and team description papers $C D$ ROM, 2006.

[22] Masayuki Mishima, Katsuhiro Hirata, and Hiroshi Ishiguro. Performance analysis of new linear actuator for android employing $3 \mathrm{~d}$ finite element method. In Proc. of The 6th International Symposium on Linear Drives for Industrial Applications, 2007.

[23] I. Noda, S. Suzuki, H. Matsubara, M. Asada, and H. Kitano. Robocup97 the first robot world cup soccer games and conferences. AI magazine, 19(3):49-59, 1998.

[24] Yoshiyuki Ohmura, Yasuo Kuniyoshi, and Akihiko Nagakubo. Conformable and scalable tactile sensor skin for curved surfaces. In Proceedings of the 2006 IEEE International Conference on Robotics and Automation (ICRA), pages 1348-1353, 2006.

[25] Peter Stone, Minoru Asada, Tucker Balch, Raffaello D'Andrea Masahiro Fujita, Bernhard Hengst, Gerhard Kraetzschmar, Pedro Lima, Nuno Lau, Henrik Lund, Daniel Polani, Paul Scerri, Satoshi Tadokoro, Thilo Weigel, and Gordon Wyeth. Robocup-2000: The fourth robotic soccer world championships. AI magazine, 22(1):11-38, 2001.

[26] Peter Stone, Tucker Balch, and Gerhard Kraetzschmar, editors. RoboCup-2000: Robot Soccer World Cup IV. Springer, Lecture Note in Artificial Intelligence 2019, 2001.

[27] Manuela Veloso, Tucker Balch, Peter Stone, Hiroaki Kitano, Fuminori Yamasaki, Ken Endo, Minoru Asada, M. Jamzad, B. S. Sadjad, V. S. Mirrokni, M. Kazemi, H. Chitsaz, A. Heydarnoori, M. T. Hajiaghai, and E. Chiniforooshan. Robocup-2001: The fifth robotic soccer world championships. AI magazine, 23(1):55-68, 2002.
[28] Manuela Veloso, Enrico Pagello, and Hiroaki Kitano, editors. RoboCup99: Robot Soccer World Cup III. Springer, Lecture Note in Artificial Intelligence 1856, 2000.

[29] Yuichiro Yoshikawa, Minoru Asada, Koh Hosoda, and Junpei Koga. A constructive approach to infant's vowel acquisition through motherinfant interaction. Connection Science, (4):245-258, 2003. 